\title{
Current Situations and Development Trends of Urban Parking Guidance and Information System
}

\author{
Xun-You NI \\ State Key Laboratory of Ocean Engineering, School of \\ Naval Architecture, Ocean \& Civil Engineering, \\ Shanghai Jiao Tong University, \\ Shanghai 200240, China \\ E-mail: nixunyou2008@sina.com
}

\begin{abstract}
In order to keep up with the latest development trends and fully absorb the reasonability of the new technologies and concepts, the current development situations were introduced and the short and long-term trends were predicted. The current development situations are that Variable Message Sign (VMS) is commonly used to release parking information. The short and long-term trends predictions are relying on the wide usage of smart phones and self-driving vehicles respectively. The technological advances facilitate the development of Parking Guidance and Information System and would promote the service level.
\end{abstract}

Keywords- intelligent transportation system; parking guidance and information system; variable message sign; smart phone; selfdriving vehicles

\section{INTRODUCTION}

With the development and application of Intelligent Transportation Systems (ITS), Parking Guidance and Information (PGIS) has been gradually implemented in large and medium-sized cities around the world. The target of PGIS is to solve the parking problem in urban areas, in which parking spaces are usually high-statured utilized. Owing to the rapid growth of private vehicles and the parking arrangement was gained the less attentions, the shortage of parking spaces has become a major transportation issue $[1,2]$. In early 1970s, PGIS was implemented in Europe and Japan. The first PGIS appeared in Aachen, Germany in 1971, and French, Swiss, and Japanese subsequently adopted the similar systems [3, 4].

In China, the planning of PGIS was started in 1990s. With introducing the concepts and technologies of ITS, Beijing and Shanghai are the first cities to construct PGIS, which achieves rather good performances. In December 2001, the first PGIS was operated in Wangfujing district, Beijing, China. Variable Message Sign (VMS) is the main way to release parking information and $51 \mathrm{VMSs}$ in total are configured to service 10 main parking lots [5]. In 2002, PGIS was constructed in Huangpu district, Shanghai, China. Locations of parking lots, travel directions, and the number of vacant spaces are the main information displayed on VMSs. The project services 18 major parking lots with more than 3000 spaces and the region starts from Middle Tibet Rd in the west and ends at Huangpu

\author{
Li-Yan CAO \\ The Third Section of Urban Planning, \\ Shenzhen Urban-space Planning and Architectural Design \\ Co., Ltd (Shanghai), \\ Shanghai 200092, China \\ E-mail: caoliyan521@163.com
}

River and starts from Suzhou River in the north and ends at East Yan'an Rd [6].

The remainder of this paper is structured as follows. Section II introduces PGIS structure and the current development situations. In Section III, with the wide usage of the smart phones, the short trend was predicted as: parking reservation system, indoor guidance, and inverse searching. Section IV predicts the long term trend mainly depending on the self-driving vehicles. Finally, conclusions were provided in Section V.

\section{CuRRENT DEVElopMENT Situations}

PGIS mainly consists of four modules: counting mechanism, control center, display terminal, and telecommunication network, taking charging of collecting, processing, releasing, and exchanging the information respectively [7]. The PGIS structure is presented in Figure 1.

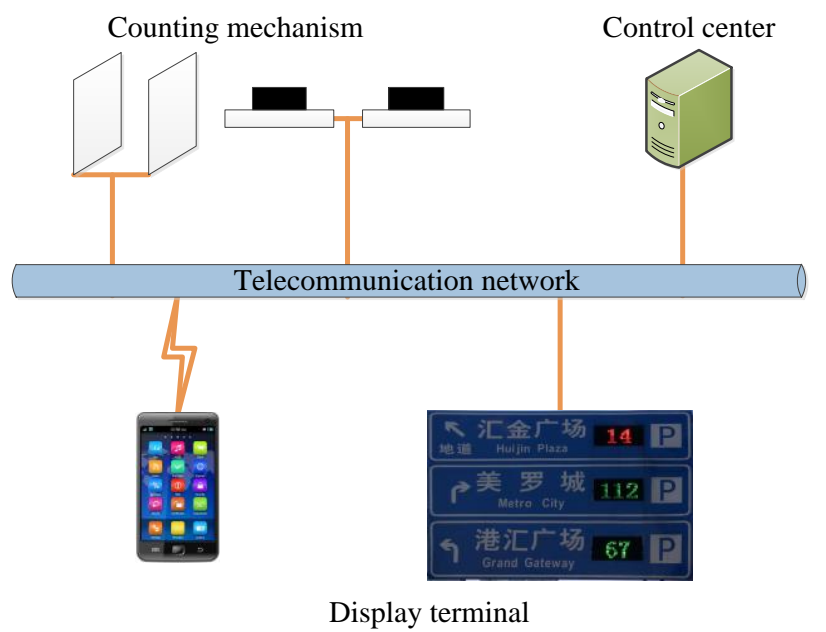

Figure 1. Basic structure of PGIS.

The counting mechanism is generally the sensors configured at the parking spaces or the entrance of parking lots. The former has the ability to monitor the availability of each space, while the latter only counts the number of arrival/departing vehicles. The control center processes the 
data transmitted from the counting mechanism and determines the content displayed on the terminals. For the vehicles with smart phones, the control center deals with the reservation requests according to the vehicular positions and speed and the parking lots availability. The display terminal consists of two main forms: VMS and smart phones. The former has been implemented in large and medium-sized cities, and the latter has good developing prospects. The connection modes of the telecommunication network are the wired and the wireless.

For different display terminals, the process algorithms adopted by the control center are critical. VMS is a common form of PGIS display terminal, which has been adopted in Europe and Japan [8-10]. In China, the terminal is also the main way to release parking information $[11,12]$. In terms of the travel distance, VMSs are divided into three levels. The first level is located at the entrance of a parking area, releasing the basic parking and traffic conditions. The second level is located along the path from the entrance of the parking area to the parking lots, displaying the detailed parking information and travel directions. The third level is located right in front of each parking lot, preventing the queue length becoming too long. Classifications and display contents of VMSs are presented in Figure 2.

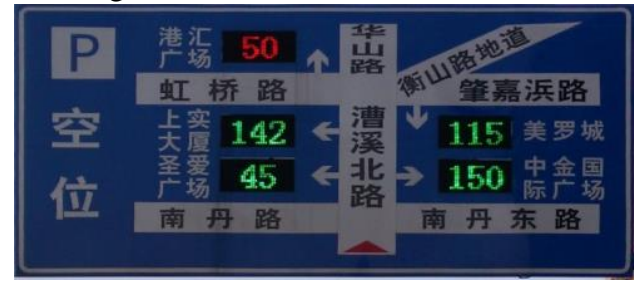

(a)

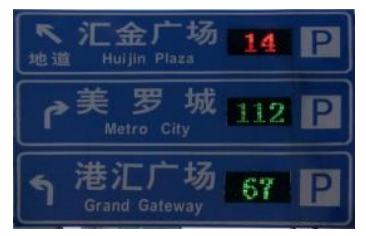

(b)

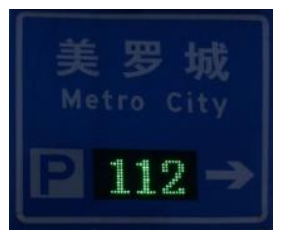

(c)
Figure 2. Classifications and display contents of VMSs: (a) the first level; (b) the second level; (c) the third level.

\section{SHORT TERM TREND PREDICTION}

In recent years, the wide usage of smart phones realizes the real-time communication between drivers and PGIS, overcoming the disadvantage VMS cannot provide parking information anywhere anytime [13]. Additionally, the smart phones can offer the personalized parking services, such as parking reservation system, indoor guidance, and inverse searching.

\section{A. Parking Reservation System}

With the assistance of the smart phones, drivers can receive the parking information and even reserve a parking space. The general rule of parking reservation is that if the target parking lot has vacant spaces, the request is accepted; otherwise, rejected. As a result, drivers would directly park their vehicles in the reserved parking lot and the travel time decreases accordingly. To manage the parking spaces, Geng and Cassandras [14] integrated the allocation center into the parking reservation system.

The process is summarized as follows: firstly, the allocation center assigns the parking spaces according to the traffic and parking conditions, such as the parking demands, the travel time, and the parking lot availability. Then, the reservation requests are dealt with: if a driver is not satisfied with the assignment results, she/he can reserve the parking space; otherwise, has to wait until the next allocation point.

\section{B. Indoor Parking Guidance}

In the urban areas, many large-sized parking lots are constructed. In the parking lots, it is difficult for drivers to identify the right travel directions. Integrating the location module, the smart phones realize the parking guidance in parking lots. The process of indoor parking guidance is presented in Figure 3.

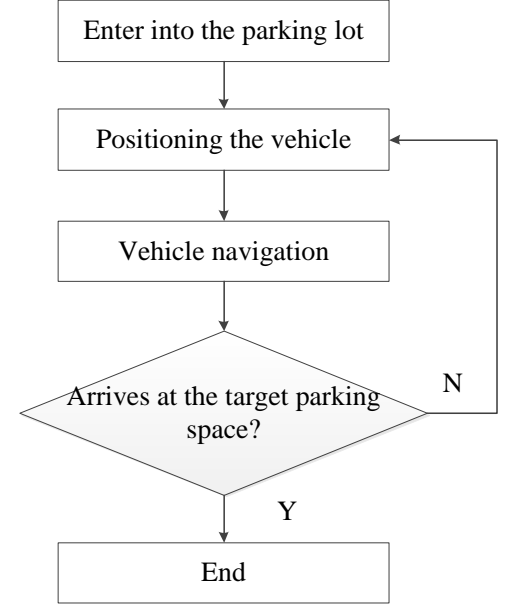

Figure 3. Process of indoor parking guidance.

The process of the indoor guidance is summarized as follows: first of all, every vehicle entering into the parking lot heads to the target space. Then, the vehicle position is obtained and the route leading to the target space is determined. Finally, examine whether the vehicle arrives at the target space, if yes, the vehicle is parked; otherwise, the process continues.

\section{Inverse Searching}

Similar to the indoor guidance, the inverse searching process is as follows: firstly, the driver position is gained and the vehicle position is known. Then, the driver accesses the route leading to the vehicle position, if he/she gets the vehicle, the process ends; otherwise, a new searching would be activated. Additionally, the running vehicles are informed to the drivers and ensure the personal security.

\section{LONG TERM TREND PREDICTION}

With the wide usage of self-driving vehicles, new requirements would be put forward for parking service. In 2010, Google started the project testing the self-driving vehicles on urban roads. Up to August 2012, the total 
kilometers of safety driving are 480 thousand, which are longer than the life cycle of the majority of ordinary vehicles [15]. In summary, the long term trend prediction mainly depends on the self-driving vehicles.

\section{A. Integrated Display Terminals}

For the self-driving vehicles, the terminals VMS and smart phones become meaningless. Without any control, steering wheel or pedal, the self-driving vehicles can stop or go relying on the sensors. The display terminals would be integrated and the activities should be directly handled by the control center, the system structure becomes more practical.

\section{B. Processing and Communication}

The self-driving vehicles will generate mountains of data, such as traffic conditions, parking information, vehicular positions and speed. For the control center, the higher processing speed is required. Additionally, the data should be transmitted more accurately. With the advance technologies in computer and communication, the requirements would be satisfied.

\section{Parking Types}

For the ordinary vehicles, the critical factor effecting parking choice is the walking distance from the target parking lot to the destination. For the self-driving vehicles, the vehicles deliver the travelers to the destinations, and then autonomously head to the target parking lot. When travelers need to use the vehicles, they inform the vehicles to come over. Consequently, the parking lots are not required to be constructed closed to the destinations and the parking spaces in front of the buildings need to increase. Additionally, because the self-driving vehicles travel is an autonomous process free from human interference, the parking reservation system would be more efficiently utilized.

As for the shared self-driving vehicles, the parking lots are not necessary in the daytime. In fact, the vehicles become one form of public transport, which are running on the urban roads. The vehicles are all public, and travelers only need to purchase the usage service. When one traveler has been delivered to the destination, the next one would wait or schedule to be picked up by the vehicles.

\section{CONCLUSIONS}

With the development and application of Intelligent Transportation Systems (ITS), Parking Guidance and Information (PGIS) has been gradually implemented in large and medium-sized cities around the world. In 1971, the first PGIS appeared in Aachen, Germany. In China, the planning of PGIS was started in 1990s. Beijing and Shanghai are the first cities to construct PGIS, which achieves rather good performances. VMS is a common form of PGIS display terminal, which has been adopted in Europe and Japan. In China, the terminal is also the main way to release parking information.

In recent years, the wide usage of smart phones realizes the real-time communication between drivers and PGIS, overcoming the disadvantage VMS cannot provide parking information anywhere anytime. Additionally, the smart phones can offer the personalized parking services, such as parking reservation, indoor guidance, and inverse searching. In the future, with the popularity of self-driving vehicles, new requirements would be put forward for parking service. For the self-driving vehicles, the display terminals would be integrated, the higher processing speed and the more accurate communication are required, and the parking spaces in front of the buildings need to increase. Additionally, in the daytime, the parking lots are not necessary for the shared self-driving vehicles.

\section{REFERENCES}

[1] S. C. Wong, C. O. Tong, W. C. H. Lam, and R. Y. Fung, "Development of parking demand models in Hong Kong," Journal of Urban Planning and Development, vol. 126, no. 2, pp. 55-74, 2000.

[2] Z. S. Qian and R. Rajagopal, "Optimal occupancy - driven parking pricing under demand uncertainties and traveler heterogeneity: A stochastic control approach," Transportation Research Part B, vol. 67, pp. 144-165, 2014.

[3] J. W. Polak, I. C. Hilton, K. W. Axhausen, and W. Young, "Parking guidance systems: Current practise and future prospects," Traffic Engineering and Control, vol. 31, no. 10, pp. 519-524, 1990.

[4] H. Z. Guan, L. H. Liu, and M. J. Liao, "Approach for planning of parking guidance and information system," Journal of Highway and Transportation Research and Development, vol. 20, no. 1, pp. 136-139, 2003. (In Chinese)

[5] L. H. Dong, "The research and application analysis for the new generation parking information service system in China," Proceedings of 6th International Conference on ITS Telecommunications, Chengdu, China, 2006.

[6] H. Zhang, "A comparative study of parking lot guidance systems at home and abroad - A case study of Cologne and Shanghai," Planners, vol. 24, no. B09, pp. 23-25, 2008. (In Chinese)

[7] P. van Der Waerden, H. Timmermans, and P. Barzeele, "Car drivers' preferences regarding location and contents of parking guidance systems: Stated choice approach,” Transportation Research Record: Journal of the Transportation Research Board, vol. 2245, pp. 63-69, 2011.

[8] A. Khattak and J. Polak, "Effect of parking information on travelers' knowledge and behavior," Transportation, vol. 20, no. 4, pp. 373-393, 1993.

[9] K. W. Axhausen, J. W. Polak, M. Boltze, and J. Puzicha, "Effectiveness of the parking guidance system in Frankfurt am Main," Traffic Engineering and Control, vol. 35, no. 5, pp. 304-309, 1994.

[10] R. G. Thompson, K. Takada, and S. Kobayakawa, "Understanding the demand for access information," Transportation Research Part C: Emerging Technologies, vol. 6, no. 4, pp. 231-245, 1998.

[11] Y. K. Mo, B. Y. Zhang, and K. F. Yan, "A study of parking behavior and parking information requirements in Shanghai CBD," Proceedings of the 7th International Conference of Chinese Transportation Professionals (ICCTP), Shanghai, China, 2007.

[12] Z. W. Liu, W. Deng, and D. W. Pan, "Driver response to parking guidance and information systems in Nanjing," Proceedings of 11th International Conference of Chinese Transportation Professionals (ICCTP), Nanjing, China, 2011.

[13] B. Zou, N. Kafle, O. Wolfson, and J. J. Lin. A mechanism design based approach to solving parking slot assignment in the information era. Transportation Research Part B: Methodological, vol. 81, pp. 631-653, 2015.

[14] Y. F. Geng and C. G. Cassandras, "New "Smart Parking" system based on resource allocation and reservations," IEEE Transactions on Intelligent Transportation Systems, vol. 14, no. 3, pp. 1129-1139, 2013.

[15] Q. L. Duanmu, J. W. Ruan, and J. Ma, "Development and advanced technology of driverless car," Agricultural Equipment \& Vehicle Engineering, vol. 52, no. 3, pp. 30-33, 2014. (In Chinese) 